\title{
Correction to: Assessment of Climate Change over the Indian Region
}

\author{
R. Krishnan, J. Sanjay, Chellappan Gnanaseelan, Milind Mujumdar, \\ Ashwini Kulkarni, and Supriyo Chakraborty
}

\section{Correction to: \\ R. Krishnan et al. (eds.), Assessment of Climate Change over the Indian Region, https://doi.org/10.1007/978-981-15-4327-2}

In the original version of the book, belated corrections as listed below are incorporated:

Chapter 1:

The caption of figure 1.5 has been changed as follows:

Spatial patterns of change in the June-to-September seasonal precipitation ( $\mathrm{mm}^{-1}{ }^{-1}$ ) over the globe in the left-hand column, and over India in the right-hand column. In the top row are plotted the observed changes for the period (1951-2014) relative to (1900-1930) over the globe based on the CRU dataset, and over India based on the IMD dataset. Plots in the middle row are from the IITM-ESM simulations for the historical period, and the plots in the last row are from the IITM-ESM projections following the SSP5-8.5 scenario. The IITM-ESM simulated changes in the historical period (first and middle rows) are plotted as difference for the period (1951-2014) relative to (1850-1900). Changes under the SSP5-8.5 scenario (last row) are plotted as difference between the far-future (2070-2099) relative to (1850-1900).

Chapter 2:

On page 40, line 5 the word "business-as-usual" has been changed to "twenty-first century under this high emission scenario".

Chapter 4:

On page 88, the last sentence "The business as usual scenario will continue to increase atmospheric $\mathrm{CO}_{2}$ and $\mathrm{CH}_{4}$ loading for next several decades." has been changed to "Without rapid mitigation policies, atmospheric $\mathrm{CO}_{2}$ and $\mathrm{CH}_{4}$ loading will continue to increase for the next several decades."

The erratum chapters and book have been updated with the changes.

The updated version of these chapters can be found at https://doi.org/10.1007/978-981-15-4327-2_1, https://doi.org/10.1007/978-981-15-4327-2_2, https://doi.org/10.1007/978-981-15-4327-2_4 